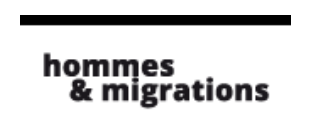

\section{Hommes \& migrations}

Revue française de référence sur les dynamiques

migratoires

$1322 \mid 2018$

Exposer les migrations

\title{
Photographier l'immigration : entre formalisme sémiologique et engagement social
}

Retour sur l'exposition itinérante Migrants en Guyane (2011-2012)

\section{Frédéric Piantoni et Katia Kukawka}

\section{(2) OpenEdition}

Journals

Édition électronique

URL : https://journals.openedition.org/hommesmigrations/6678

DOI : $10.4000 /$ hommesmigrations. 6678

ISSN : 2262-3353

Éditeur

Musée national de l'histoire de l'immigration

Édition imprimée

Date de publication : 1 juillet 2018

Pagination : 101-111

ISBN : 978-2-919040-42-1

ISSN : 1142-852X

Référence électronique

Frédéric Piantoni et Katia Kukawka, «Photographier l'immigration : entre formalisme sémiologique et engagement social », Hommes \& migrations [En ligne], 1322 | 2018, mis en ligne le 01 juillet 2020, consulté le 21 janvier 2022. URL : http://journals.openedition.org/hommesmigrations/6678 ; DOI : https://doi.org/10.4000/hommesmigrations.6678 


\section{PHOTOGRAPHIER L'IMMIGRATION : ENTRE FORMALISME SÉMIOLOGIQUE ET ENGAGEMENT SOCIAL RETOUR SUR L'EXPOSITION ITINÉRANTE MIGRANTS EN GUYANE (2011-2012)}

Par FRÉDÉRIC PIANTONI, maître de conférences en géographie, université de Reims, UMR $196 \mathrm{CePeD}$ (université Paris-Descartes, IRD), et KATIA KUKAWKA, conservatrice en chef du patrimoine, directrice adjointe du musée d'Aquitaine.

\section{La compréhension et l'exposition des réalités migratoires} en Guyane par le prisme de la photographie constituent la force et l'originalité de Chercher la vie. Migrants en Guyane, une exposition itinérante créée par le Musée des cultures guyanaises de Cayenne en 2011. Cette démarche photographique visait à représenter le quotidien vécu par les migrants en Guyane. II s'agissait donc de trouver la juste distance pour donner à voir leurs parcours de vie dans leur environnement. Ce questionnement sur l'instant de la prise de vue et l'espace de représentation des sujets conduit à envisager la photographie à l'aune d'une sémiologie globale nourrie à la fois d'histoire de l'art et de géographie sociale.

En 2011, le Musée des cultures guyanaises saisissait l'opportunité d'une Année des Outre-mer français pour présenter, dans trois villes de l'Hexagone, l'exposition Chercher la vie. Migrants en Guyane. Le projet, conçu à partir de la sélection d'une cinquantaine de photographies de Frédéric Piantoni, répondait aux ambitions de cette Année : «Donner de la lisibilité aux cultures ultramarines, dans toute leur diversité et leur richesse $e^{1}$. » Il visait aussi à donner à voir les plus «invisibles » de la société guyanaise : des hommes et des femmes en migration, venus chercher la vie sur ce territoire français d'Amérique du Sud où, aujourd'hui encore, un habitant sur deux n’y est pas né. 


\section{Légendes des photos d'ouverture}

Photo 1. Les boîtes aux lettres au bas des collines sont caractéristiques d'un habitat non planifié. Une part importante de la population immigrée ne dispose pas de documents de régularisation : aucun accès à l'emploi, aux prêts, au foncier. Par conséquent, l'informalité devient la norme, mais ne signifie pas une absence de règle. Les quartiers immigrés se nichent en haut des collines ou dans des zones de marais, difficiles d'accès. La gestion des quartiers dépend d'une approche collective, quasi-coutumière, en marge des règles d'urbanisme ou des plans de prévention des risques (glissement de terrain, inondations). Ainsi, on observe des concentrations de boîtes aux lettres correspondant à des noms, mais dont l'habitation reste aussi invisible qu'indésirable.

Photo 2. Début d’après-midi, les membres se retrouvent pour la pause méridienne. La construction sur pilotis, puis à l'aide de taule légère permet à l'habitat de se situer au-dessus des fréquentes inondations que subit cette zone insalubre en raison d'un drainage déficient. Le quartier de la Matinha est construit sur une ancienne décharge et reste une espace non constructible aux yeux du plan d'urbanisme.

Photo 3. Les couleurs pimpantes et la scène des femmes en manucure montrent que la vie n'est pas misérable malgré l'environnement d'un bidonville que l'on perçoit par les déchets aux sols. L'humain, dans ce paysage urbain, donne ici une temporalité qui fait aussi le lien avec le reportage social.

La Région Guyane, collectivité de tutelle du Musée des cultures guyanaises, avait en 2009 validé les grandes orientations d'un nouveau projet scientifique et culturel (PSC) pour son musée, implanté depuis les années 1990 dans une petite maison créole du centre de Cayenne. Ce PSC dessinait une nouvelle institution ambitieuse à une dizaine de kilomètres du centre-ville de Cayenne, à proximité immédiate des vestiges de l'une des plus anciennes habitations coloniales de la Guyane $e^{2}$. Le conseil scientifique du musée avait été profondément remanié et sa nouvelle composition souhaitait témoigner d'une volonté d'ouverture vers quelquesuns des grands enjeux de la Guyane contemporaine : ses liens avec les États voisins du Suriname et du Brésil, le plurilinguisme, la question migratoire ${ }^{3}$. Quant au comité de pilotage du projet, il était présidé par Jean-Paul Fereira, vice-président de la région en charge de la culture, mais aussi maire d'une commune frontalière de l'ouest guyanais fortement imprégnée d'une histoire migratoire ancienne et plus récente ${ }^{4}$.

Le comité de pilotage et le conseil scientifique considéraient ensemble la nécessité pour le futur musée de traiter du fait migratoire dans toute sa complexité et sa profondeur historique. Une nécessité impérieuse, au vu des fantasmes et des crispations auxquels renvoient presque systématiquement toutes les discussions à son sujet : «les migrants » seraient ainsi responsables de tous les maux de la Guyane contemporaine, et particulièrement d'une insécurité désormais structurelle, tant en ville (Cayenne, Kourou et Saint-Laurent-du-Maroni) que sur les routes (les deux nationales du littoral et les deux grandes « routes » fluviales que sont l'Oyapock et le Maroni).

\section{Penser l'exposition}

En tant que commissaire scientifique (Frédéric Piantoni) et commissaire technique (Katia Kukawka) de l'exposition, nous avons voulu aller à l'encontre de ce discours uniforme sur les " migrants », tel qu'il se donne à lire dans les pages des faits divers de la presse guyanaise. Les éléments scientifiques rassemblés depuis une quinzaine d'années le permettaient. Diverses recherches que nous avions menées sur les réseaux transnationaux, l'accès à l'habitat et à l'emploi, les transferts

2. Profondément remanié en 2012, ce projet devrait finalement trouver place dans l'ancien hôpital colonial de Cayenne, qui fait aujourd'hui l'objet d'importants travaux de rénovation. 3. Cinq chercheurs composaient le conseil scientifique du MCG en 2009 Isabelle Hidair (Université de Guyane), Lucia Hussak van Velthem (SCUP/MCT, Brasilia), Isabelle Léglise (CNRS), Frédéric Piantoni (Université de Reims/IRD), Thomas Polimé (anthropologue indépendant, Pays-Bas). 4. La guerre civile au Suriname, qui vit affluer plusieurs milliers de réfugiés dans les années 1980. 
financiers, ont constitué la matière de plusieurs rapports et publications scientifiques au cours des quinze dernières années. Par ailleurs, nous disposions d'un matériau inédit et conservé avec soin : des portraits en noir et blanc de celles et de ceux rencontrés au fil des ans sur un site d'orpaillage, dans une rue de Cayenne et dans un quartier d'habitat illégal, sur une place de marché et près du fleuve. Autant de visages d'immigrés en Guyane, rassemblés entre 2005 et 2009.

L'objectif de l'exposition était de donner la parole aux immigrés, reflétant leur individualité, la singularité de leur parcours. Les matériaux rassemblés le permettaient puisqu'à chaque portrait correspond un récit, fruit de longs entretiens menés en face-àface - la prise de vue n'arrivant qu'à l'issue de la parole confiée. Un catalogue viendra compléter l'exposition, avec un propos développé sur la condition migratoire en Guyane ${ }^{5}$.

Le premier chantier a consisté à composer et à organiser une sélection parmi les centaines de clichés. Ce travail a été mené avec les représentants de deux organismes finançant le projet : le Parc amazonien de Guyane et la Direction des affaires culturelles de Guyane. Quatre thématiques ont rapidement émergé : les quartiers, les femmes, les parcours et les frontières. À ce travail préalable ont succédé les recherches de financements, de lieux d'accueil dans l'hexagone et d'un éditeur pour le catalogue. Cette étape s'est avérée relativement simple, à partir du moment où le projet a été labellisé "Année des outre-mer » et a donc obtenu la garantie d'une bonne visibilité. Le budget global du projet - 138000 euros - était notable pour une exposition produite en Guyane; il a permis de réaliser ce que nous espérions : un dispositif d'exposition itinérante parfaitement autonome, pouvant s'adapter à des configurations spatiales très diverses ne nécessitant rien d'autre qu'une alimentation électrique. Techniquement, il fallait concevoir et organiser l'exposition à distance, en métropole, soit à
8000 kilomètres de la Guyane, notre lieu de résidence. La conception de la scénographie a ainsi dû être confiée à un prestataire métropolitain, plus à même de suivre toutes les phases de fabrication et d'accompagner les montages et démontages successifs. Après consultation restreinte, nous avons retenu la proposition de Renaud Morel, designer-scénographe installé près de Lyon, qui imagine habituellement des cabanes perchées dans les arbres. De longues et nombreuses séances de travail à distance ont peu à peu donné vie au projet, destiné à occuper successivement sept lieux d'exposition.

Le premier chantier a consisté à composer et à organiser une sélection parmi les centaines de clichés. Ce travail a été mené avec les représentants de deux organismes finançant le projet : le Parc amazonien de Guyane et la Direction des affaires culturelles de Guyane. L'exposition a finalement été présentée en 2011 à la Cité de la bande dessinée d'Angoulême, puis à la Bibliothèque universitaire de La Rochelle et à la Cité nationale de l'histoire de l'immigration, avant de traverser l'Atlantique pour rejoindre, en 2012, le Camp de la transportation (Saint-Laurent du Maroni) puis l'EnCRE (Ensemble culturel régional de Cayenne). Soit un total cumulé de près de onze mois de présentation, entre l'Hexagone et la Guyane, pour une fréquentation totale difficile à évaluer : l'exposition était en accès libre sur les cinq sites et seule la Cité de la bande dessinée a réalisé un comptage assez fin du public (estimé à plus de 10000 personnes).

\section{La photographie dans le corpus de recherche en géographie sociale}

Le travail photographique exposé s'est inscrit tôt dans la pratique de recherches et d'analyses menées dans le cadre de programmes relatifs à l'histoire, aux mémoires et aux circulations migratoires en Guyane $^{6}$. Les méthodologies utilisées ont permis de 
définir des catégories de migrants et d'espace dans lesquels ils évoluent. Ainsi, la photographie dans le projet Migrants en Guyane n'est pas un outil anthropologique, mais une ressource intégrée dans un corpus plus large qui n'avait pas vocation à être donnée à voir, à être exposée.

Les images constituent une extraction et résument des situations formelles d'individus dans un environnement de réussite ou d'échec. Elles sont l'aboutissement d'une chaîne d'opérations de recherche : le traitement de bases de données statistiques, l'échantillonnage, puis les enquêtes, l'approfondissement et l'élargissement par des entretiens, enfin les biographies migratoires appartenant aux catégories et aux groupes sociaux les plus représentés. Les documents photographiques en formalisent le portrait social (individu) ou urbain (quartier d'immigration) en situations de migration.

Cependant, ces photographies sont aussi des documents qui s'inscrivent dans une démarche d'engagement social à l'occasion des élections régionales de 2010. Cette période était marquée par des tensions xénophobes, des discriminations à

l'égard des migrants, des discours

Le travail photographique exposé s'est inscrit tôt dans

la pratique de recherches et d'analyses menées dans le cadre de programmes relatifs à l'histoire, aux mémoires et aux circulations migratoires en Guyane. identitaires et sécuritaires, et de l'instrumentalisation politique, prémices aux mouvements sociaux de 2017. Tous ces phénomènes restent encore très vivaces aujourd'hui. En effet, la faiblesse de l'information sur la situation des migrants laissait le champ libre à la démagogie et aux propos extrêmes. Elle interrogeait le rôle du chercheur dans la construction d'un « principe partagé, universel, qui ferait des migrants, plutôt qu'un problème, une cause pour tous, au sens d'une épreuve qui nous tire en avant vers la compréhension et le désir d'un monde commun ${ }^{7}$ ». L'objectif était de faire connaître le quotidien d'une réalité migratoire, en dressant un portrait en miroir des indicateurs finalement déshumanisants et confortables dans leur mobilisation par les acteurs publics. Cette complémentarité a permis d'éviter un stérile débat communautaire qu'une approche anthropologique aurait immanquablement attisé au regard de l'ethnicisation des rapports sociaux en Guyane.

Cette approche par une géographie sociale, mettant l'accent sur l'inscription spatiale et économique des migrants dans les marges urbaines guyanaises, pouvait articuler la situation vécue par les migrants avec ceux qui les côtoyaient et ne percevaient qu'une spécificité économique ou sociale. Ce faisant, ce corpus d'images associé à un discours, tantôt vulgarisé, tantôt sensible, a permis de proposer des catégories migratoires différentes des stéréotypes reconnaissables et discriminants. En somme, de donner corps à une aventure humaine, plus qu'à un discours sur l'immigration.

\section{La construction de l'image: entre formalisme sémiologique et engagement social}

Si le fond d'informations procède de la rigueur scientifique, il en est de même pour la forme retenue dans la composition et la sémiologie : le choix du noir et blanc pour des portraits en plan américain, ou de la couleur pour les prises de vue panoramiques des paysages urbains des quartiers des migrants. Il s'agit de montrer la part du concret et de l'indicible que révèle l'aventure humaine. Dans cette perspective, nous avons mobilisé une démarche héritée du photojournalisme et d'un ancrage dans le reportage social.

Icône française du photojournalisme, Henri CartierBresson (1908-2004), dans le seul texte qu'il ait écrit sur sa pratique photographique, note que le traitement d'un sujet suppose que les « (...) rapports de forme doivent être rigoureusement établis. On doit situer son appareil dans l'espace par rapport à l'objet, et là commence le grand domaine de la composition. La photographie est pour moi la reconnaissance dans la réalité d'un rythme de surfaces, de lignes ou de valeurs ; l'œil découpe le sujet et l'appareil n'a qu'à faire son 
travail, qui est d'imprimer sur la pellicule ${ }^{8}$ la décision de l'œil ${ }^{9} »$. Cette véritable éducation du regard ressort d'une analyse fine des formes spatiales et de leurs échelles d'appréhension ; une approche géographique, en somme.

En complément de la forme, l'engagement vis-à-vis du sujet est primordial. La recherche d'une problématique a pour conséquence d'explorer des sujets peu ou pas traités, des mondes nouveaux et des « terrains » socio-spatiaux peu balisés. Le fond et la forme sont valorisés en photographie et en géographie. Ils impliquent pour le photojournaliste, comme pour le géographe, un rapport presque fusionnel à son espace de recherche, son « terrain ». La compréhension qu'il en a est totalisante (incluant aussi bien le milieu et que la société), à l'image de Joël Bonnemaison, Edmond Bernus, Pierre Gourou, Pierre Monbeig etc. Cela a prévalu en géographie de la fin du XIX ${ }^{e}$ siècle jusqu'aux années 1960-1970. Pierre-Arnaud Chouvy relevait ainsi que si « la photographie et la géographie enjoignent à la découverte, elles encouragent aussi la proximité : avec le terrain, l'objet, le sujet. Robert Capa (1913-1954), illustre photographe de guerre et fondateur en 1957 de Magnum Photos avec Henri CartierBresson et d'autres, expliquait que si une photo n'était pas bonne c'est que le photographe nétait pas assez rapproché de son sujet ${ }^{10} »$.

\section{La formalisation de l'esthétisme géo-photographique au début du XXe siècle}

Dans la lignée de Charles Nègre (1820-1880) ${ }^{11}$, précurseur en la matière, le photographe américain

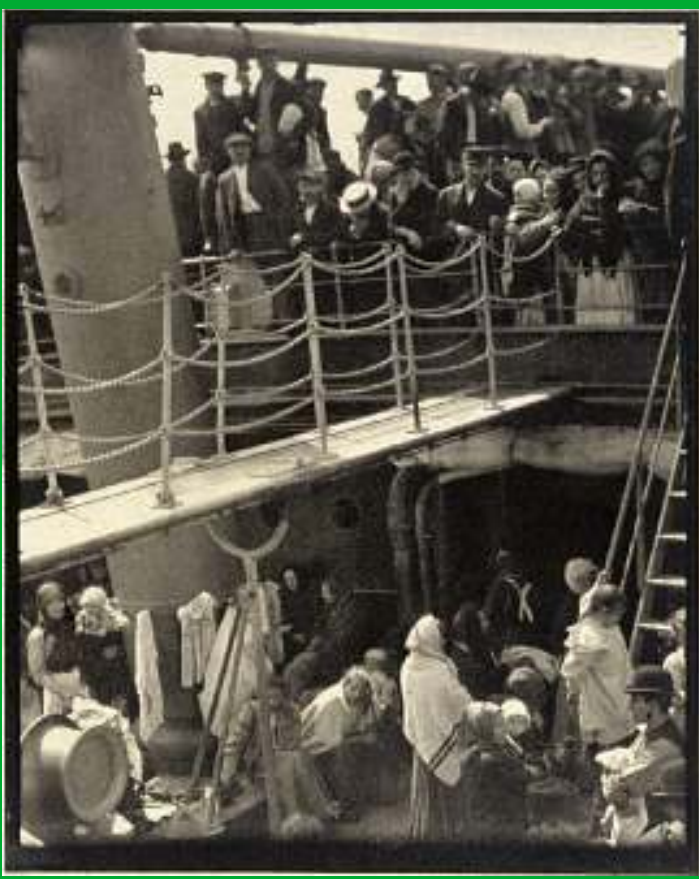

Alfred Stieglitz, The Steerage 1911, prise de vue de 1907 Epreuve photomécanique (photogravure) sur papier japon. Format 33,2 × 26,3 cm. Source : Paris, Musée d'Orsay. (c) ADAGP, PARIS - RMN-GRANd PALAIS (MUSÉE D'ORSAY).

Alfred Stieglitz (1864-1946) érige la photographie comme un art à part entière. En 1902, il fonde le groupe Photo-Secession, issu de la scission du groupe pictorialiste, considérant la photographie comme un art analogue à la peinture. Il fonde la revue Camera Work (1903-1917), support de promotion de cette nouvelle conception de la photographie tournée vers des sujets non académiques. Ce

8. Note : la pellicule de format $35 \mathrm{~mm}$ (support gélatino-argentique ou multicouches chromatiques) a été supplantée par les capteurs numériques. Cependant, ce support est encore utilisé pour la photographie instantanée en noir et blanc, ainsi que dans les grands formats des chambres photographiques $(9 \times 12 \mathrm{~cm}, 20 \times 25 \mathrm{~cm}, 50 \times 60 \mathrm{~cm})$. 9. Gabriel Bauret, Approches de la photographie, Paris, Armand Colin, 2004, p. 42. 10. Pierre-Arnaud Chouvy, "Tropisme géographique et tropisme photographique ", in EspacesTemps.net, 30 juin 2008. 11. Ce photographe français, artiste-peintre de formation, s'est attaché à photographier les petits métiers des rues de Paris, s'inspirant d'une tradition établie de longue date en gravure et en peinture. Mais, alors que ces sujets sont traités de manière statique, il les photographie en action. 12. Littéralement photographie directe, débarrassée des artifices. 
courant, dit de la Straight Photography ${ }^{12}$, s'ouvre sur le documentaire social, puis le photojournalisme qui en constitue la forme la plus aboutie. Un esthétisme photographique nouveau naît, qui est fondé sur l'instantané de la prise de vue d'une part, et qui évacue l'ensemble des techniques et des règles de composition classique jugées proches de la peinture d'autre part.

Une photographie symbolise cette rupture, The Steerage (L'entrepont), prise en 1907 et publiée en 1911. A. Stieglitz la considère comme une image novatrice associant la forme et le fond : "La scène m'a fasciné : Un chapeau de paille rond ; l'entonnoir penchéà gauche, l'escalier penchéà droite ; le pont-levis blanc, ses rampes en chaîne; des bretelles blanches croisées sur le
Alfred Stieglitz (1864-1946) érige la photographie comme un art à part entière. En 1902,

il fonde le groupe PhotoSecession, issu de la scission du groupe pictorialiste, considérant la photographie comme un art analogue à la peinture. dos d'un homme en bas; des machines circulaires en fer ; un mât qui coupe le ciel, complétant le triangle. J'ai été envoûté durant un moment. J'ai vu des formes liées entre elles - une image de ces formes, et sous-jacente, une nouvelle vision qui me retenait: des gens simples; le sentiment $d u$ bateau, de l'océan, du ciel (..... ${ }^{13}$. » Selon Richard Whelan, biographe de Stieglitz, cette prise de vue a été réalisée durant une croisière de l'Amérique vers l'Allemagne ${ }^{14}$. On l'interprète souvent comme le retour dans leur pays des personnes refoulées par les États-Unis ${ }^{15}$. Si le cliché est pris en 1907, Alfred Stieglitz ne s'y intéresse vraiment qu'à partir de 1911, le publiant dans le numéro 36 de Camera Work (Institut des arts de Minneapolis, 2008). Il saisit l'entassement des classes populaires sur un paquebot. L'image n'est pas recadrée. Différents éléments du décor, une cheminée, une échelle et une passerelle, structurent l'image autour de la tache de lumière que constitue le chapeau rond et blanc de l'un des passagers.

Alfred Stieglitz a donc tracé une voie nouvelle. Désormais, les photographes présentent leurs œuvres comme des miroirs du monde, des reflets auxquels leurs commentaires donnent du sens : « Le motif fait la photo, le modèle dicte l'image ${ }^{16}$. » Dans l'entre-deux-guerres, ces documentaristes ont fourni les témoignages les plus durables sur l'Allemagne de Weimar, à l'image d'August Sander ${ }^{17}$, et sur l'Amérique en crise des années 1935-1943. Durant cette période, les photographes de la Farm Social Administration ${ }^{18}$, comme Dorothea Lange (18951965), Ben Shahn (1898-1969) ou Walker Evans (1903-1975), ont enregistré et prodigué une interprétation iconographique des effets des crises écologique et économique aux États-Unis (Dust Bowl et Grande Dépression). Par leur engagement social, par leur proximité forte avec leur sujet, et par la manière de composer une photogénie de la misère, les images eurent un impact considérable. Elles permirent aussi de rallier l'opinion publique aux réformes du New Deal de Franklin Delano Roosevelt ${ }^{19}$. Enfin, diffusées dans tout le pays en raison de la gratuité des droits de publication, ces images contribuèrent à la connaissance de leur pays pour un grand nombre de citoyens étasuniens ${ }^{20}$.

13. Alfred Stieglitz cite par Weston Naef (dir.), In Focus: Alfred Stieglitz: Photographs from the J. Paul Getty Museum, Los Angeles, J. Paul Getty Museum Publications, 1995, p. 44. 14. Richard Whelan, Stieglitz on Photography: His Selected Essays and Notes, New York, Aperture, 2000, p. 197. 15. Toutefois, si des passagers pourraient avoir été refoulés en raison de leur incapacité de satisfaire les exigences financières ou de santé pour l'entrée sur le territoire, il est plus probable que la plupart d'entre eux soient des artisans ayant travaillé dans le secteur de la construction, en plein essor à l'époque. En effet, des travailleurs hautement qualifiés dans l'artisanat, tels que l'ébénisterie, le travail du bois, et le marbre ne se voyaient attribuer que des visas temporaires de deux ans. Voir Richard Whelan, op. cit. 16. Olivier Lugon, Le style documentaire. D'August Sander à Walker Evans 1920-1945, Paris, Macula, 440 pages, 2011 [2001], 114 photographies. 17. Photographe allemand (1876-1964). Son œuvre immense et inachevée repose sur des portraits de la société allemande par catégories socioprofessionnelles (l'artisan, le paysan etc.). Son travail associe documentaire social et esthétisme formel alors que les sujets participent à leurs propres portraits. 18. La Farm Social Administration (FSA) a été créée en 1937, dans le cadre du New Deal, cette agence est chargée de réformes et d'aides à l'agriculture pour les paysans les plus pauvres. Afin de promouvoir son travail et de faire passer ses réformes, l'organisme se dote d'un service de communication et d'illustration, sous la direction d'un visionnaire en la matière Roy E. Stryker (1893-1975). II recrute une douzaine de photographes connus pour leur engagement social et politique. Ils sont chargés de documenter, aussi objectivement que possible, les conditions de vie des agriculteurs du pays. Des images deviendront des symboles, connus de tous et largement diffusés. L'agence photographique de la FSA devient l'Office of War Information de 1942 à 1943, son action s'achevant avec le New Deal. 19. Gilles Mora, Beverly W. Brannan, Les photographies de la FSA Farm Social Administation. Archives d'une Amérique en crise 1935-1943, Paris, Seuil, 460 photographies en bichromie, 2006, p. 20. 20. Ibid. 
À partir des années 1950, de nombreux photographes vont prolonger cette œuvre alors que les progrès techniques et la géopolitique des années 1950-1990 projettent les photojournalistes sur la scène mondiale des conflits de la décolonisation et de la guerre froide. Entre géographie et photographie, des magazines comme Life, Vu, Paris Match, National Geographic ou Géo délivrent une représentation des territoires et des sociétés produites par l'information. La fonction de la photographie est celle du témoignage en utilisant les codes sémiologiques hérités de la Straight Photography. Le photojournalisme a contribué à assimiler le photographe à un explorateur, un arpenteur, le témoin privilégié des lieux où le monde change. Cette image rejoint une vulgate assimilant le géographe à celui qui trace, inscrit, cartographie, découvre et témoigne lui aussi.

\section{Les portraits sociaux de l'exposition: entre photojournalisme et rencontre de l'autre}

Comprendre un "monde nouveau », en l'occurrence celui du migrant, implique une approche lente et une immersion dans le groupe social qu'il étudie et dont il veut dégager une interprétation conforme aux données, c'est-à-dire en étudiant comment une variable statistique se lit dans le mode de vie.

Décrivant ce processus, la photographe étatsunienne Diane Arbus (1923-1971) ${ }^{21}$ remarquait : « Si jétais simplement curieuse, il me serait très difficile de dire à quelqu'un: "J'ai envie de venir chez vous, et de vous faire parler, de vous faire raconter l'histoire de votre vie." Je veux dire que les gens vont me répondre: "Vous êtes cinglée." De plus, ils vont être sur leurs gardes. Mais l'appareil photo est une sorte de passepartout. Chez beaucoup de gens, il y a une envie quion leur accorde une certaine attention ${ }^{22}$. "

La restitution d'une scène impliquant des individus nécessite l'instauration d'une confiance réciproque. En effet, l'action de déclencher, rapide et incisive, rend nécessaire l'importance d'une relation bien comprise avec les sujets photographiés.
Le photojournalisme a contribué à assimiler le photographe à un explorateur, un arpenteur, le témoin privilégié des lieux où le monde change. Un mot ou une attitude ( $y$ compris dans la façon de se saisir de l'appareil) peuvent rompre le crédit instauré ; le photographe, et partant le géographe, donne alors l'impression d'être un insupportable intrus. L'attente et la compréhension des implicites sont nécessaires pour capter le récit et composer la photographie.

Margaret Bourke-White (1904-1971), photojournaliste étasunienne dont l'œuvre est marquée par le reportage social, illustre l'importance de cette capacité à entrer en relation en étant très attentif à l'attitude: "Parfois, je montais l'appareil dans un coin de la pièce, je masseyais à une certaine distance avec un déclencheur souple à la main, et je regardais nos gens pendant que $M$. Caldwell leur parlait. Il pouvait se passer une heure avant que leurs visages ou leurs gestes nous offrent ce que nous étions en train d'essayer d'exprimer, mais à l'instant même où cela se produisait, la scène était fixée sur la pellicule avant même qu'ils ne sachent ce qu'il s'était passé $e^{23} . "$

L'importance de "figer la scène » rejoint ce que Brassai ${ }^{24}$ notait : "Le monde n'a de signification que dans l'instant ${ }^{25} »$. Le photographe entendait par là que la composition d'une photographie montrait 


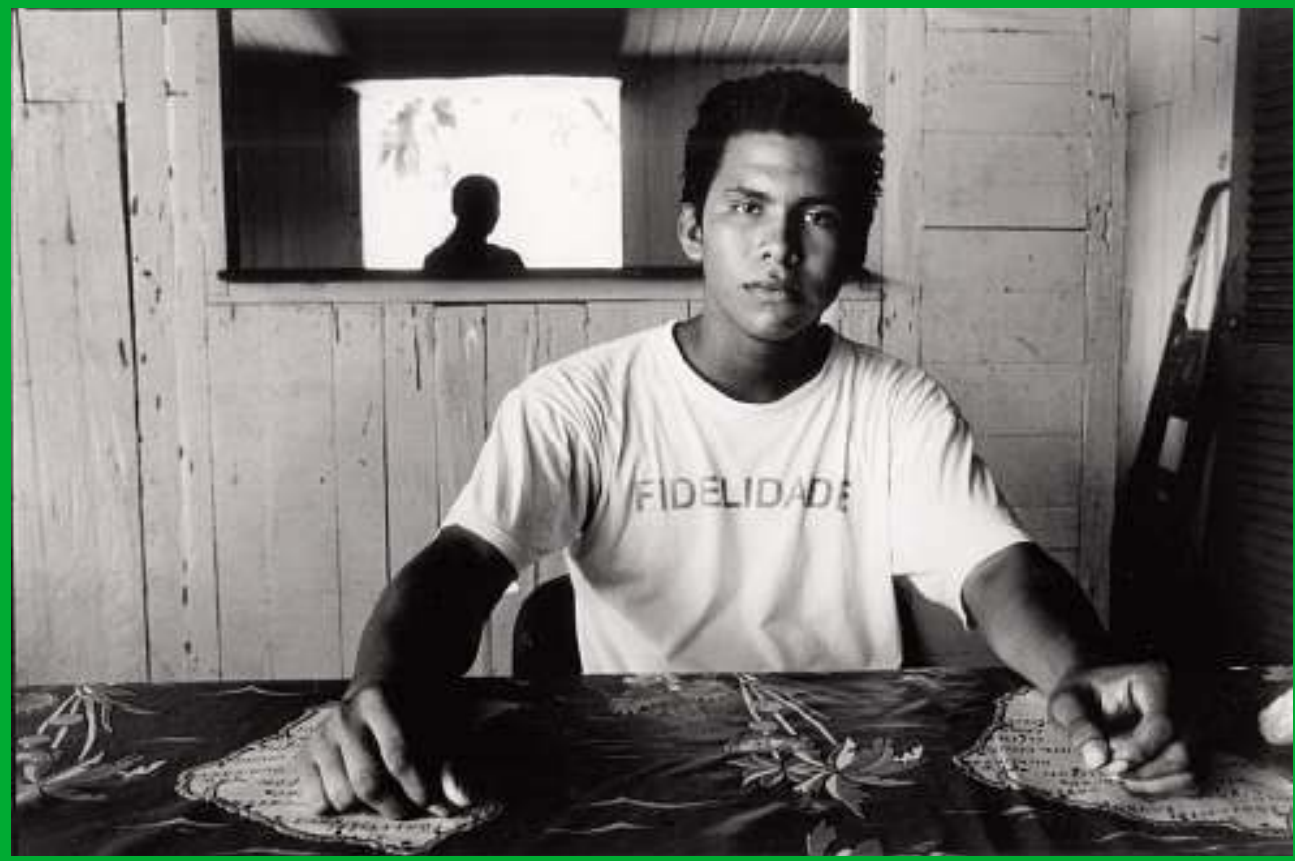

Adolescent brésilien, membre de l'Assembleira de Deus, dans le local de l'église Assembleira de Deus, quartier Mathina, Cayenne, 2010. Tirage gelatino-argentique, format $30 \times 40 \mathrm{~cm}$. @ FRÉDÉRIC PIANTONI, 2008.

un " monde » singulier, une synthèse dans l'espace et dans le temps, formalisée dans l'instant du mouvement de l'obturateur.

La photographie ci-dessus incarne le propos. Ce cliché date de 2008. Il a été pris dans le local de l'église Assembleira de Deus, dans le bidonville de Matinha à Cayenne, où loge une population majoritairement brésilienne. Au cours du second entretien consacré au parcours migratoire de cet adolescent de 17 ans, fils du pasteur, la discussion porte, au moment précis de la prise de vue, sur l'importance du rôle social des obédiences religieuses dans les quartiers précarisés. Cette photographie s'inscrit dans une série de cinquante portraits d'immigrés ${ }^{26}$. Les images se veulent narratives, esthétiques et en lien avec un commentaire contextualisé. En captant des aspects d'une vie sociale en mouvement, la restituant par une composition (précision du cadrage, plan américain) et une technique (lumière, profondeur de champ, mouvement, texture), les individus figurent selon un point de vue valorisant, communiquant une empathie par l'expression d'un visage, d'un regard. L'arrière-plan informe sur l'environnement de réussite ou de précarité sociale de l'individu. Pourquoi déclencher à ce moment? L'adolescent, revenant sur son parcours, exprimait l'importance des valeurs transmises par son église (regard et flocage 
Fidelidade sur le maillot) et l'importance du nombre de fidèles en Guyane (les deux poings symboliquement posés sur la toile cirée aux motifs de la Guyane), alors que son père, le pasteur, apparut au troisième plan comme une ombre hitchcockienne semblant manipulatrice ou inquiétante (perspective à l'angle fort de l'image). À ce moment précis, la manière d'interpréter la scène s'effectue en déclenchant; l'esthétisme de la forme rejoint le signifié.

Henri Cartier-Bresson conceptualise l'action de saisir la scène comme l'« instant décisif ». Au cours de la situation photographiée, l'équilibre de l'image, le rapport du temps avec l'espace relèvent « de la reconnaissance simultanée, dans une fraction de seconde, d'une part de la signification d'un fait, et de l'autre de l'organisation rigoureuse des formes perçues visuellement qui exprime ce fait ${ }^{27}$ ». Ce moment ne se reproduira jamais et, en ce sens, cette image prend la dimension d'une interprétation événementielle, une forme de témoignage dans un cadre spatial et temporel figé (daté), "une mince tranche d'espace autant que de temps » pour reprendre l'expression de Susan Sontag ${ }^{28}$.

Un autre exemple, tiré d'une situation dans un tissu urbain dense, montre l'importance de l'emplacement et des objets entrant dans la composition $^{29}$. Ce paysage urbain est constitué à partir de six photographies prises avec un pied photographique, puis assemblées a posteriori sous la forme d'un panoramique. Cette technique renforce les perspectives tout en évitant les déformations qu'induirait l'emploi d'un objectif grand-angulaire. La structure graphique est fondée sur les rythmes verticaux (porte, barrières, poteaux de fil à linge) et horizontaux (linteaux, travées sur pilotis et planches de la maison violette) constitués par l'architecture précaire des matériaux de récupération. La photographie est découpée en scénettes. Les couleurs pimpantes et la scène des femmes en manucure (soin du corps) montrent que la vie n'est pas misérable malgré l'environnement d'un bidonville que l'on perçoit par les déchets aux sols. L'humain, dans ce paysage urbain, donne ici une temporalité qui fait aussi le lien avec le reportage social.

\section{De la représentation à l'abstraction}

Ainsi, plus on serait proche du sujet, plus il s'en dégagerait des images qui le décrivent avec pertinence. Pourtant, on peut aussi considérer que la photographie, vue comme un acte fidèle de reproduction, n'a pas de sens : le cadre est d'emblée une contrainte qui enchâsse une situation dans un espace à deux dimensions. L'utilisation du noir et blanc ou des gammes chromatiques en est une autre et, enfin, l'instantanéité de la prise de vue rend caduque toute conformité avec une réalité à dupliquer. La photographie est donc un artefact au sens d'un phénomène créé de toutes pièces par les conditions expérimentales.

Par ailleurs, on ne perçoit que ce que l'on est prêt à voir ; le choix des éléments qui vont composer l'image est tributaire de cette Par ailleurs, on ne perçoit que ce que l'on est prêt à voir ; le choix des éléments qui vont composer l'image est tributaire de cette lacune. L'image n'est qu'une part d'un argumentaire décrivant une scène analysée.

d'un argumentaire décrivant une scène analysée. Elle n'est donc, en rien, un témoignage ou une preuve, souvent avancée comme telle, mais une somme d'éléments graphiques agencés et choisis par l'auteur à des fins de descriptions ; une photographie est une impression représentée plus ou moins abstraite, plus ou moins composée, plus ou moins lisible.

L'auteur peut justifier sa démarche et définir les références qui ont présidé à la réalisation de cette œuvre-scène (spatiale) à ce moment précis (une attitude, une lumière, un angle de vue, un rythme graphique, etc.). Il peut développer son sujet ou laisser le spectateur sans information, lui laissant mener sa propre analyse de l'image en fonction de 
sa propre connaissance du sujet. Finalement, une photographie ne dit rien du lieu ni du contexte dans lesquels elle est prise. Pour l'écrivain et sémiologue français Roland Barthes (1915-1980), ce que la photographie "reproduit à l'infini n'a eu lieu qu'une fois ; elle répète mécaniquement ce qui ne pourra jamais plus se répéter existentiellement ${ }^{30} »$. Ainsi, il n'y a pas de photo sans quelque chose ou quelqu'un. La photo est invi-
À l'image de l'hybridité des catégories observées dans les analyses migratoires, il serait pertinent de multiplier les apports graphiques, plastiques, picturaux enrichissant les situations migratoires que nous avons traitées. sible, ce n'est pas elle qu'on voit mais l'objet ${ }^{31}$. «La photographie ne dit pas (forcément) ce qui n'est plus, mais seulement et à coup sûr, ce qui a été. Cette subtilité est décisive. Devant une photo, la conscience ne prend pas nécessairement la voie nostalgique $d u$ souvenir, mais pour toute photo existant au monde, la voie de la cer-

titude : l'essence de la Photographie est de ratifier ce qu'elle représente $(. . .)^{32}$.»

La géographe et photographe Anaïs Marshall rappelle ainsi que "l'objectivité voulue du géographe, à travers ses productions scientifiques (cartes, entretiens, photographies), est essentielle. Il est couramment mentionné que l'on peut faire dire ce que l'on souhaite à une carte, il en va de même pour une photographie. L'angle de vue, le cadrage, la couleur, la luminosité de la prise, le sujet, l'assemblage, etc. influencent l'interprétation ${ }^{33}$ ».

De fait, l'image et la photographie sont, par définition, "polysémiques » en ce sens qu'elles ont la faculté d'avoir plusieurs significations et de se prêter à plusieurs interprétations ${ }^{34}$.

Pourtant si la représentation évoquée ici est tributaire d'une réalité concrète dont le photographe saisit un focus, d'autres photographies se fondent sur une reconstruction virtuelle et abstraite du réel, à partir de la composition organisée d'objets multiples (Andréas Gursky ou Jeff Wall, par exemple).
Comme en géographie, les aspects descriptifs illustrés par l'image conduisent à une abstraction de l'interprétation: une conceptualisation des structures de l'espace a priori. Ainsi, l'apparition du support numérique et la croissance des capacités de traitement informatique permettent un travail sur de grands formats et l'insertion de multiples éléments dans l'image. Les œuvres sont conçues et réalisées à partir d'une problématique définie à l'avance. En somme, la représentation de la réalité est réinterprétée pour en réaliser une autre qui, bien que virtuelle, n'en est pas moins conforme à des situations existantes ; une modélisation fidèle, dont les structures sont logiquement appariées et s'enrichissent d'apports permettant de porter un discours cohérent sur l'image.

À l'image de l'hybridité des catégories observées dans les analyses migratoires, il serait pertinent de multiplier les apports graphiques, plastiques, picturaux enrichissant les situations migratoires que nous avons traitées. De fait, entre création artistique et démarche de recherche, la frontière est poreuse. C'est aussi ce que l'exposition Migrants en Guyane s'est attachée à montrer, en se jouant des catégorisations pour convoquer le sensible et l'empathie.

\section{Conclusion : photographier n'est pas assez}

Photographier n'est pas assez, choisir un format, composer, styliser les postures, intégrer le sujet par le jeu des plans, saturer des couleurs ou jouer des ombres révèlent partiellement l'acuité du regard et sa part invisible. Pas assez, car les émotions tributaires de la mémoire des expériences visuelles et affectives n'apparaissent pas dans le champ de prime abord. Il s'agit de savoir comment les suggérer par une sémiologie globale, alors que les 
courants photographiques sont bousculés par une diversité de styles et de pratiques attachées à des codes mondialisées ou trop singuliers pour être partagés, et, enfin, que les supports numériques autorisent des traitements diversifiés et des temporalités de diffusion très courtes.

Bien davantage qu'un éparpillement, qu'un nivellement ou qu'une transgression, ces apports autorisent des registres d'expressions aussi riches qu'hybrides. Aussi, seules comptent les façons de nous émouvoir et de témoigner par une iconographie formalisant une réalité épurée, abstraite ou reconfigurée, mais toujours inscrite comme une référence.

Étudier les migrations nous enjoint à dépasser les dogmatismes et les catégories fonctionnelles a priori, à comprendre l'essence des relations à l'altérité, à l'exil, à l'intégration, à l'incroyable aventure humaine. Photographier l'immigration pousse, de même, à une originalité plastique et graphique au service d'un propos exigeant et engagé. 